\title{
Augmenting B with Control Annotations
}

\author{
Wilson Ifill ${ }^{1,2}$ \\ Steve Schneider ${ }^{1}$ \\ Helen Treharne ${ }^{1}$ \\ ${ }^{1}$ Department of Computing, University of Surrey and ${ }^{2}$ AWE Aldermaston
}

\begin{abstract}
CSP $\| \mathrm{B}$ is an integration of the process algebra Communicating Sequential Processes (CSP), and the B-Method, which enables consistent controllers to be written for B machines in a verifiable way. Controllers are consistent if they call operations only when they are enabled. Previous work has established a way of verifying consistency between controllers and machines by translating control flow to AMN and showing that a control loop invariant is preserved. This paper offers an alternative approach, which allows fragments of control flow expressed as annotations to be associated with machine operations. This enables designers' understanding about local relationships between successive operations to be captured at the point the operations are written, and used later when the controller is developed. Annotations provide a bridge between controllers and machines, expressing the relevant aspects of control flow so that controllers can be verified simply by reference to the annotations without the need to consider the details of the machine operations. This paper presents the approach through two instances of annotations with their associated control languages, covering recursion, prefixing, choice, and interrupt.
\end{abstract}

\section{Introduction}

The design and implementation of critical systems benefits from development in a formal method such as the B-Method, which models systems in terms of state and operations. However, this approach does not support specifications of execution patterns directly, and so approaches such as Event-B [MAV05] and CSP $\| \mathrm{B}$ [ST05] have been proposed to incorporate action specification with B. This paper develops the CSP $\| \mathrm{B}$ approach, which offers a clean separation of control from data manipulation. The developments presented here fall within the scope of AWE's System-B project, which involves collaborative research into the use of CSP $\| \mathrm{B}$ to specify co-designs [MS06] and to formally investigate systems designs of large scale developments.

One motivation for the work is a desire to enable Engineers to describe many aspects of design within a single notation. We introduce control annotations into the B-Method to enable the formal capture of control flow fragments in 
$\mathrm{B}$ during the development of the B machines. We generate proof obligations to demonstrate that the set of executions allowable by the annotations do not cause operations to diverge. The benefit of this approach is that only the semantics of the machine operations is required in checking the annotations, and these checks are similar in size and difficulty to standard B machine consistency checks. Annotations can be checked against controllers written in CSP, which describe the flow of control explicitly. There is no need to check the CSP directly against the full B description, in contrast to previous CSP $\| \mathrm{B}$ work where it was necessary to translate the entire CSP controller into AMN in order to check it. Once the annotations are shown to be correct with respect to the $\mathrm{B}$ machine we can evaluate controllers against the annotations without further reference to the machine.

This paper describes the extendable framework for introducing annotations and controllers and presents two exemplars. In Section 2, we briefly introduce the approach. In Section 3 we demonstrate the framework by using a simple language for controllers, the NEXT annotation for B operations, and define the notion of consistency between them. Section 4 presents a worked example of a simple traffic control system. In Section 5 we introduce an interrupting annotation FROM-ANY, add the CSP interrupt operator to the controller language, and extend the notion of consistency. We develop the worked example in Section 6 to illustrate the new annotation and its use. Finally in Section 7 we discuss further directions and related work.

We assume the reader is familiar with the Abstract Machine Notation of the BMethod [Abr96]. We restrict our attention in this paper to correct B machines: those for which all proof obligations have already been discharged. We use $I$ to refer to the invariant of the machine, $T$ to refer to the machine's initialisation, $P_{i}$ to refer to the precondition of operation $O p_{i}$, and $B_{i}$ to refer to the body of operation $O p_{i}$.

Controllers will be written in a simple subset of the CSP process algebraic language [Hoa85,Sch99]. The language will be explained as it is introduced. Controllers are considered as processes performing events, which correspond to operations in the controlled B machine. Thus operation names will appear in the controller descriptions as well as the B machine definitions.

\section{The general framework}

The approach proposed in this paper introduces annotations on B operations as a mechanism for bridging the gap between B machines and CSP controllers, whilst maintaining the separation of concerns. The approach consists of the following components:

- Machine definition: the controlled component must first be defined. 


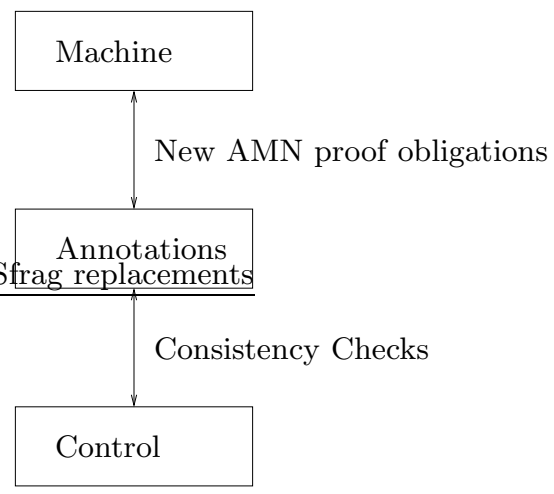

Fig. 1. Relationship between the different parts of the approach

- Annotations: the initialisation and the operations in the machine definition are annotated with fragments of control flow.

- Annotation proof obligations: verification conditions that establish consistency of the annotations with the controlled machine. This means that the fragments of control flow captured by the annotations really are appropriate for the machine.

- Controller: this is a process that describes the overall flow of control for the B machine.

- Consistency checking: establishing that the controller is consistent with the annotations - that every part of the control flow is supported by some annotation.

Checking a CSP controller against a machine is thus reduced to checking it against the annotations and verifying that the annotations are appropriate for the machine. The relationship between the different parts of the approach is illustrated in Figure 1.

The framework presented here is quite general, in that it may be applied to a variety of annotations and control languages. The first step to be taken is therefore to fix on the control language and the associated annotations to be incorporated into the AMN machine descriptions. The key result that these build up to is expressed in Theorem 1, though the underlying theory will not be expanded in this paper for reasons of space.

\section{A first approach}

We will demonstrate the approach firstly with a simple model to illustrate how the aspects of the approach interrelate. The first kind of annotation we consider is the NEXT annotation, and we use an extremely simple controller language 
consisting only of prefixing, choice, and recursion. These go naturally together because the NEXT annotation is concerned with successive operations, and the controller language allows simple loops of sequences of operations.

\subsection{The NEXT annotation}

We annotate an operation of a B machine with a NEXT annotation. Currently, we introduce this as a comment included with the description of the operation, so that it is invisible to current tools. However, in principle tools could be modified to recognise an additional ANNOTATION clause to introduced the additional information into operation descriptions.

A NEXT annotation on an operation $O p_{i}$ introduces another operation $O p_{j}$, or set of operations $O p_{j}, \ldots, O p_{k}$, which should be enabled after $O p_{i}$ is executed.

The NEXT annotation is written as follows:

$$
O p_{i} \widehat{=} \mathbf{P R E} P_{i} \text { THEN } B_{i} \text { END } / *\left\{O p_{j}, \ldots, O p_{k}\right\} \mathrm{NEXT} * /
$$

\subsection{Annotation proof obligations}

The annotation corresponds to the assertion that, following the execution of $O p_{i}$, operations $O p_{j}$ through to $O p_{k}$ are available for execution. This gives rise to the following proof obligation, which requires that the precondition of each of the listed operations is enabled:

Definition 1 (NEXT Proof Obligation for Operations). The proof obligation associated with a NEXT annotated operation $O p_{i}$ is given as:

$$
\begin{aligned}
& \left(I \wedge P_{i} \Rightarrow\left[B_{i}\right]\left(P_{j}\right)\right) \\
\wedge & \ldots \\
\wedge & \left(I \wedge P_{i} \Rightarrow\left[B_{i}\right]\left(P_{k}\right)\right)
\end{aligned}
$$

If the conjunction of proof obligations for all the annotations are discharged then we say that the annotations are consistent with the machine. This ensures that any controller which only calls operations that are listed, following execution of $O p_{i}$, can be sure that those operations will be enabled.

Definition 2 (NEXT Proof Obligation for Initialisation). The proof obligation associated with the annotation

$T / *\left\{O p_{j}, \ldots, O p_{k}\right\} N E X T * /$ 
on initialisation $T$ is given by

$$
\begin{aligned}
& {[T]\left(P_{j}\right) } \\
\wedge & \ldots \\
\wedge & {[T]\left(P_{k}\right) }
\end{aligned}
$$

This establishes that all of the listed operations are enabled following initialisation. Thus, any controller which only begins with such operations will be consistent with the controller.

We will use $n \operatorname{ext}\left(O p_{i}\right)$ to identify the set of operations given in the NEXT annotation. Thus from the annotation above we have that $\operatorname{next}\left(O p_{i}\right)=\left\{O p_{j}, \ldots, O p_{k}\right\}$. We also use next(INITIALISATION) to identify the set of operations in the annotation of the INITIALISATION clause. To ensure that there is no deadlock in the system, every operation, and the INITIALISATION, must have a NEXT annotation.

\subsection{A simple controller language}

We will begin with the following simple controller language, which allows only event prefix, choice, and recursion:

\section{Definition 3 (Controller Syntax).}

$$
R::=a \rightarrow R|R \square R| S
$$

Here, the event $a$ is an operation name, and $S$ is a process variable. Recursive definitions are then given as $S \widehat{=} R$. In a controller definition, all process variables used are bound by some recursive definition. The results presented in this paper require that all recursive definitions are guarded, which means that at least one event must occur before a recursive call.

We can now give a definition of consistency between a controller and the annotations on a B machine. The key underlying idea is that whenever one event $O p_{j}$ follows another $O p_{i}$ in the controller's execution, then there must be an annotation that underpins this, ensuring that the associated operation $O p_{j}$ is guaranteed to be enabled after $O p_{i}$ has occurred.

To do this, we first capture the initial events init $(R)$ for a controller $R$ :

Definition 4 (initial elements of CSP controller process).

$$
\begin{aligned}
\operatorname{init}(a \rightarrow R 1) & =\{a\} \\
\operatorname{init}(R 1 \square R 2) & =\operatorname{init}(R 1) \cup \operatorname{init}(R 2) \\
\operatorname{init}(S) & =\operatorname{init}(R) \quad \text { where } S \widehat{=} R
\end{aligned}
$$

Note that in a controller definition the process variable $S$ must be bound by some recursive definition $S \widehat{=} R$, and this defines $\operatorname{init}(S)$.

For example, if $L O O P \widehat{=} a \rightarrow b \rightarrow L O O P$, then $\operatorname{init}(L O O P)=a$. 


\subsection{Consistency}

A controller will be step-consistent with a collection of annotations if all consecutive events are allowed by the occurrence of some annotation. In the case where the only kind of annotation is NEXT, it is straightforward to define stepconsistency, and we do this over the structure of the syntax.

Definition 5. [Step-consistency of NEXT Annotated Machines and Controllers] The step-consistency of a controller $R$ with the annotations of machine $M$ is defined structurally over the syntax of $R$ as follows:

1. $a \rightarrow R$ is step-consistent with $M$ 's annotations if init $(R) \subseteq \operatorname{next}(a)$ and $R$ is step-consistent with $M$ 's annotations.

2. $R 1 \square R 2$ is step-consistent with $M$ 's annotations if $R 1$ is step-consistent with $M$ 's annotations and $R 2$ is step-consistent with $M$ 's annotations.

3. $S$ is step-consistent with $M$ 's annotations.

A family of recursive definitions $S \widehat{=} R$ is step-consistent with $M$ 's annotations if each $R$ is step-consistent with $M$ 's annotations.

There is one additional aspect of consistency required: that the initial state of the machine is consistent with the starting point of the controller. This is captured as initial-consistency:

Definition 6 (Initial-Consistency of NEXT Annotated Machines and Controllers). A controller $R$ is initially-consistent with the annotations of machine $M$ if $\operatorname{init}(R) \subseteq \operatorname{next}($ INITIALISATION $)$.

Definition 7 (Consistency). A controller $R$ is consistent with the annotations of machine $M$ if it is step-consistent with $M$ 's annotations and initiallyconsistent with $M$ 's annotations.

The main result of this section is the following theorem:

Theorem 1. If $R$ is consistent with the annotations of a machine $M$, and the annotations of $M$ are consistent with machine $M$, then operations of $M$ called in accordance with the control flow of $R$ will never be called outside their preconditions.

The key feature of the proof of this theorem is an argument that no trace of $R$ leads to an operation of $M$ called outside its precondition. This is established by building up the traces of $R$ and showing that at each step a an operation called outside its precondition cannot be introduced, by appealing to the relevant annotation and applying its proof obligation. 


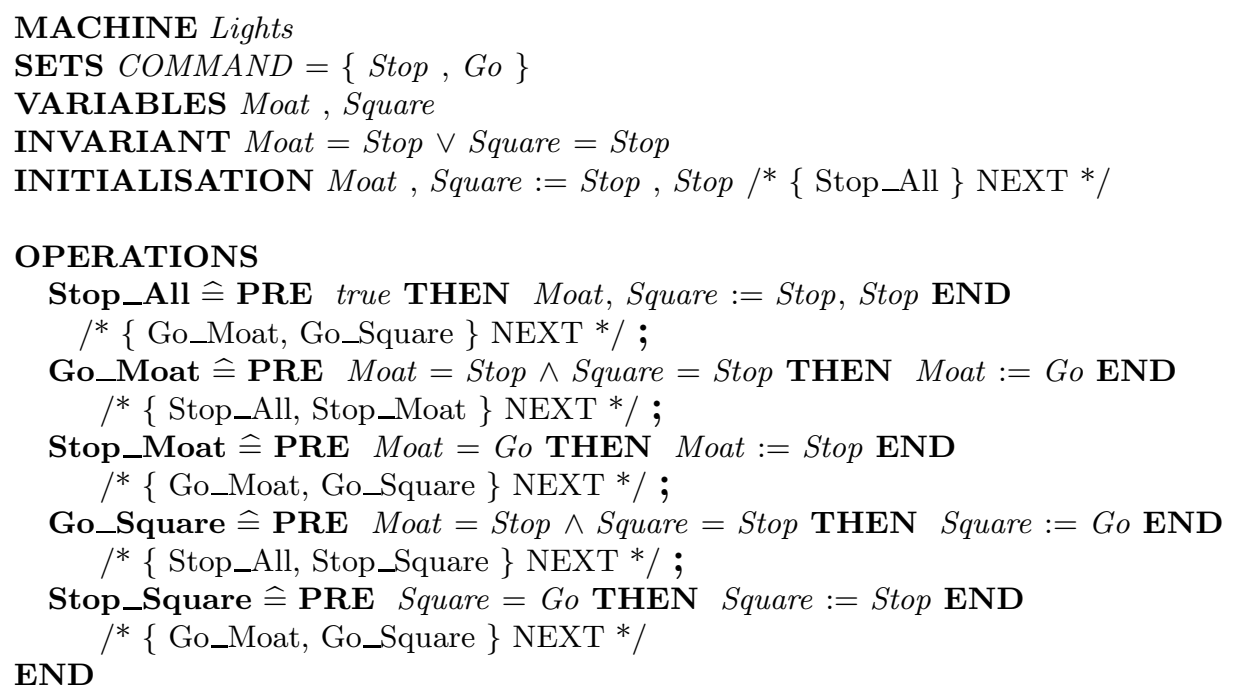

Fig. 2. Lights machine

$$
\begin{aligned}
& \text { Lights_CTRL } \widehat{=} S t o p \_A l l \rightarrow S \_C T R L \\
& \text { S_CTRL } \widehat{=}(\text { Go_Moat } \rightarrow \text { Stop_Moat } \rightarrow \text { S_CTRL }) \\
& \square(\text { Go_Square } \rightarrow \text { Stop_Square } \rightarrow \text { S_CTRL })
\end{aligned}
$$

Fig. 3. Lights Controller

The benefit of this theorem is that the details of the operations of $M$ are required only for checking the consistency of the annotations, and are not considered directly in conjunction with the controller. The annotations are then checked against the controller using the definition of consistency above. This enables a separation of concerns, treating the annotations as an abstraction of the $\mathrm{B}$ machine.

\section{Example: Carcassonne Traffic Control System}

We use the example of a traffic light system to illustrate the ideas introduced in the previous section.

A traffic control system for the main street of the walled Cité of Carcassonne is specified. The main street is narrow and is heavily used by tourists and some motor vehicles brave enough to edge through the alley. The system must allow traffic up into the cité market square from the moat or down from the square 
to the moat gate along the same single width road. The system must allow time for motor vehicles to clear the road before changing direction. A B machine that offers a choice between the traffic flows is given in Figure 2. A controller consistent with the annotations is given in Figure 3. We note that the controller specified in the annotations is a subset of the allowable actions.

In order to show that Lights_CTRL is an appropriate controller for Lights, we make use of the annotations. We must show that the annotations are consistent with the machine, and we must also show that the controller is consistent with the annotations. We consider each of these in turn.

\subsection{Consistency of annotations with the machine}

The proof obligations associated with the annotations (eliding the invariant) are as follows:

- Initialisation: the initialisation clause must establish the precondition of all the operations identified in its annotation; in this case this is Stop_All, with precondition true. From Definition 2, we must prove

$[$ Moat, Square $:=$ Stop, Stop $]($ true $)$.

- Stop_All: there are two next operations, Go_Moat and Go_Square, and so there will be a proof obligation associated with each of them. In fact each of them have the same precondition: Moat $=$ Stop $\wedge$ Square $=$ Stop. Hence the two proof obligations are identical, and correspond to

$I \wedge P_{\text {Stop_All }} \Rightarrow[$ Moat, Square $:=$ Stop, Stop $]($ Moat $=$ Stop $\wedge$ Square $=$ Stop $)$.

- Go_Moat: there are two next operations, Stop_All and Stop_Moat, identified in the annotation. For Stop_All, the precondition is true, so the proof obligation is $I \wedge P_{G o \_M o a t} \Rightarrow[M o a t:=G o]($ true $)$. Considering Stop_Moat, its precondition is Moat $=G o$, so the corresponding proof obligation is

$$
I \wedge P_{\text {Go_Moat }} \Rightarrow[\text { Moat }:=\text { Go }](\text { Moat }=\text { Go }) \text {. }
$$

- Go_Square: the annotation and hence the proof obligations for this operation are entirely similar to those for Go_Moat, but assigning to Square this time.

- Stop_Moat: there are two next operations, Go_Moat and Go_Square, so there will be a proof obligation associated with each. The proof obligation associated with Go_Moat is given by $I \wedge P_{\text {Stop_Moat }} \Rightarrow\left[B_{\text {Stop_Moat }}\right]\left(P_{\text {Go_Moat }}\right)$, which expands to

$$
\begin{aligned}
& (\text { Moat }=\text { Stop } \vee \text { Square }=\text { Stop }) \wedge(\text { Moat }=\text { Go }) \\
& \Rightarrow[\text { Moat }:=\text { Stop }](\text { Moat }=\text { Stop } \wedge \text { Square }=\text { Stop })
\end{aligned}
$$

The proof obligation associated with Go_Square is entirely similar, since the precondition for Go_Square is the same as that of Go_Moat. 
- Stop_Square: the annotation and hence the proof obligations for this operation are entirely similar to that for Stop_Moat.

In all cases the proof obligations are discharged. Note that in the case of Stop_Moat the invariant and its precondition are necessary for establishing that the operation body establishes the precondition of the next operations.

Discharging the proof obligations means that the annotations correctly provide information concerning permitted successions of operations, and can be used to verify the appropriateness of the controller.

\subsection{Consistency of the controller with the annotations}

To show that the controller Lights_CTRL is consistent with Lights we apply the definitions of step-consistent and initially-consistent. Let $R_{-} C T R L$ be the body of the definition of $S_{-} C T R L$. Then it is necessary to show that $R \_C T R L$ is step-consistent with the annotations of the Lights machine.

Step-consistency is established by considering the parts of the definition of R_CTRL:

- The process variable $S \_C T R L$ is step-consistent, by the definition of stepconsistency for process variables.

- Stop_All $\rightarrow$ S_CTRL: the prefix rule for step-consistency from Definition 5 requires that $i n i t\left(S \_C T R L\right) \subseteq n e x t\left(S t o p \_A l l\right)$. This is true in this case, since the process variable $S_{-} C T R L$ is step-consistent and

$$
\operatorname{init}\left(S \_C T R L\right)=\left\{G o \_M o a t, G o \_S q u a r e\right\}=\operatorname{next}\left(S t o p \_A l l\right) .
$$

- Stop_Moat $\rightarrow S \_C T R L:$ this is step-consistent, since the process variable $S \_C T R L$ is step-consistent and

$$
\operatorname{init}\left(S \_C T R L\right)=\left\{G o \_M o a t, G o \_S q u a r e\right\}=\operatorname{next}\left(S t o p \_M o a t\right) .
$$

- Go_Moat $\rightarrow$ Stop_Moat $\rightarrow$ S_CTRL: step-consistency follows from the fact that $S t o p \_M o a t \rightarrow S \_C T R L$ is step-consistent, and

$$
\begin{aligned}
& \operatorname{init}\left(S t o p \_M o a t \rightarrow S \_C T R L\right)=\left\{S t o p \_M o a t\right\} \\
& \subseteq\{\text { Stop_Moat,Stop_All }\} \\
& =\operatorname{next}\left(G_{0} \_ \text {Moat }\right) \text {. }
\end{aligned}
$$

- Stop_Square $\rightarrow$ S_CTRL: this is step-consistent, since the process variable $S_{-} C T R L$ is step-consistent, and

$$
\operatorname{init}\left(S \_C T R L\right)=\{\text { Go_Moat }, \text { Go_Square }\}=\operatorname{next}(\text { Go_Square }) \text {. }
$$


- Go_Square $\rightarrow$ Stop_Square $\rightarrow$ S_CTRL: step-consistency follows from the fact that the process Stop_Square $\rightarrow S_{-} C T R L$ is step-consistent, and

$$
\begin{aligned}
& \text { init }(\text { Stop_Square } \rightarrow \text { S_CTRL })=\{\text { Stop_Square }\} \\
& \subseteq\{\text { Stop_Square, Stop_All }\} \\
& =\text { next }(\text { Go_Square }) \text {. }
\end{aligned}
$$

- Go_Moat $\rightarrow$ Stop_Moat $\rightarrow S \_C T R L \square G o \_S q u a r e \rightarrow S t o p \_S q u a r e \rightarrow S \_C T R L:$ this is step-consistent, due to the step-consistency of both sides of the choice.

Initial-consistency follows from the fact that

$n \operatorname{ext}($ INITIALISATION $)=\operatorname{init}($ Lights_CTRL $)$.

Thus, S_CTRL is consistent with the annotations of the machine Lights, and so the controller is appropriate for the machine.

\section{Introducing FROM-ANY annotations and interrupts}

Section 2 introduced the key components of the annotation approach that provide a framework for developing controlled systems. In general there will be a variety of annotations that we will want to make use of, and a richer language for controllers. These will have an impact on the consistency relationship, and on the underlying proofs which will need to be adapted to accommodate the changes.

In this section we will extend the controller language to include interrupts, which are commonly used in control flow descriptions. A further annotation will be introduced to accompany this extension to the controller language, and we will see the impact on the notion of consistency.

\subsection{The From-ANY Annotation}

The introduction of interrupts in the control language gives rise to another annotation, the FROM-ANY annotation.

The FROM-ANY annotation is written / ${ }^{*}$ FROM-ANY * $/$. This annotation is added to an operation which can follow any previous operation (including itself), and can also follow initialisation. It will naturally be used on an operation which follows an interrupt, since such an operation might follow any previous operation, allowing for the fact that the operation might happen anywhere.

Its use in an arbitrary operation $O p_{i}$ is given as follows:

$O p_{i} \widehat{=} \mathbf{P R E} P_{i}$ THEN $B_{i}$ END $/ *$ FROM-ANY */; 


\subsection{Annotation proof obligation}

The annotation corresponds to the claim that after the execution of any operation, $O p_{i}$ will always be available to execute. The annotation gives rise to the following proof obligation: that the precondition $P_{i}$ of $O p_{i}$ is enabled after any precondition, and also that it is enabled after initialisation:

Definition 8 (FROM-ANY Proof Obligations). The proof obligation associated with a FROM-ANY annotated operation $\mathrm{Op}_{i}$ is given as:

$$
\begin{aligned}
& \forall o p \in O P E R A T I O N S \bullet P_{o p} \wedge I \Rightarrow\left[B_{o p}\right] P_{i} \wedge \\
& {[T] P_{i}}
\end{aligned}
$$

A condition sufficient to establish the proof obligation of Definition 8 is the assertion $I \Rightarrow P_{i}$. Its use is captured as a lemma:

Lemma 1. If $I \Rightarrow P_{i}$ for an operation $O p_{i}$ with a FROM-ANY annotation, then the proof obligations on $\mathrm{Op}_{i}$ associated with this annotation are all true.

When it holds, this is a simpler condition to establish. However, it may not always hold, since it is stronger than the FROM-ANY proof obligations.

Operations can be annotated with both a FROM-ANY annotation and a NEXT annotation. The former indicates what the operation can follow, and the latter indicates what can come next.

For a machine $M$, we define from-any $(M)$ to be the set of operations of $M$ that are annotated with a FROM-ANY clause.

\subsection{Controller language}

We introduce an interrupt operator to the control language as follows:

\section{Definition 9 (Controller Syntax).}

$$
R::=a \rightarrow R|R \square R| R \triangle R \mid S
$$

The global interrupt operator, $\triangle$, permits the second controller fragment to interrupt the former at any point, even before the first action of the former has been performed. However, we do not rely on an interrupt establishing initialconsistency. There should always be a next annotation in the INITIALISATION.

The $\operatorname{init}(R)$ function was defined on controllers by means of a structural induction over the controller syntax. Thus the introduction of an interrupt clause into the controller syntax necessitates a revision to the definition of init $(R)$, as follows: 


\section{Definition 10 (init on CSP controller process).}

$$
\begin{aligned}
\operatorname{init}(a \rightarrow R 1) & =\{a\} \\
\operatorname{init}(R 1 \square R 2) & =\operatorname{init}(R 1) \cup \operatorname{init}(R 2) \\
\operatorname{init}(R 1 \triangle R 2) & =\operatorname{init}(R 1) \cup \operatorname{init}(R 2) \\
\operatorname{init}(S) & =\operatorname{init}(R) \quad \text { where } S \widehat{=} R
\end{aligned}
$$

The first event that can be performed by $R 1 \triangle R 2$ is either a first event from $R 1$, or else a first event from $R 2$ following the occurrence of the interrupt.

\subsection{Consistency}

We again provide a definition for consistency between a controller and the annotations. This is again separated into a notion of step-consistency, which is concerned with successive events; and initial-consistency, regarding the initial state of the system.

The notion of step-consistency now needs to take account of a further clause in the controller language, and the fact that the machine $M$ has more than one kind of annotation. Thus the definition has one additional clause, and from-any $(M)$ will also appear.

Definition 11 (Step-consistency of NEXT and FROM-ANY Annotated Machines and Controllers). The step-consistency of a controller $R$ with the annotations of machine $M$ is defined structurally over the syntax of $R$ as follows:

1. $a \rightarrow R$ is step-consistent with $M$ 's annotations if $\operatorname{init}(R) \subseteq(n \operatorname{ext}(a) \cup$ from-any $(M))$ and $R$ is step-consistent with $M$ 's annotations.

2. $R 1 \square R 2$ is step-consistent with $M$ 's annotations if $R 1$ is step-consistent with $M$ 's annotations and $R 2$ is step-consistent with $M$ 's annotations.

3. $R 1 \triangle R 2$ is step-consistent with $M$ 's annotations if $R 1$ is step-consistent with $M$ 's annotations, $R 2$ is step-consistent with $M$, and init $(R 2) \subseteq$ from-any $(M)$.

4. $S$ is step-consistent with $M$ 's annotations.

A family of recursive definitions $S \widehat{=} R$ is step-consistent with $M$ 's annotations if each $R$ is step-consistent with $M$ 's annotations.

In the case for $a \rightarrow R$, we require that every operation $b$ that $R$ can perform first, which are those operations in $\operatorname{init}(R)$, must be able to follow $a$, either because $b$ is in $\operatorname{next}(a)$ and hence identified explicitly as an operation that can follow $a$, or because $b$ is in from-any $(M)$, and hence can follow anything.

In the case for interrupt, we have that $R 1$ must be step-consistent with $M$ because all executions of $R 1$ are possible executions of $R 1 \triangle R 2 ; R 2$ must also be step-consistent since control can pass to $R 2$; and every operation that $R 2$ can 


$$
\begin{aligned}
& L i g h t s \_C T R L 2 \widehat{=} S t o p \_A l l \rightarrow S \_C T R L 2 \\
& S \_C T R L 2 \widehat{=} \text { S_INNER } \triangle S t o p \_A l l \rightarrow S \_C T R L 2 \\
& \text { S_INNER } \widehat{=}(\text { Go_Moat } \rightarrow \text { Stop_Moat } \rightarrow \text { S_INNER }) \\
& \square(\text { Go_Square } \rightarrow \text { Stop_Square } \rightarrow \text { S_INNER })
\end{aligned}
$$

Fig. 4. A Second Lights Controller

initially perform must be able to follow anything, since the interrupt can occur at any point.

The cases for choice and for recursion are similar to the previous version of step-consistency.

Definition 12 (Initial-Consistency of NEXT and FROM-ANY Annotated Machines and Controllers). A controller $R$ is initially-consistent with the annotations of machine $M$ if init $(R) \subseteq \operatorname{next}($ INITIALISATION $) \cup$ from-any $(M))$.

As stated previously, a controller $R$ is consistent with the annotations of a $\mathrm{B}$ machine $M$ if it is step-consistent and initially-consistent with the annotations of $M$.

Once again we have everything in place to establish the main theorem of this section:

Theorem 2. If $R$ is consistent with the annotations of a machine $M$, and the annotations of $M$ are consistent with machine $M$, then operations of $M$ called in accordance with the control flow of $R$ will never be called outside their preconditions.

\section{Example continued}

We develop the example of the Carcassonne traffic control system. We wish to extend the controller so that normal operation can be interrupted at any point with all lights being set to Stop. The resulting controller is given in Figure 4.

The NEXT annotations of the machine Lights are not sufficient to establish consistency with Lights_CTRL2, and in particular the interrupt requires consideration. The only event immediately following the interrupt is Stop_All, so we require a FROM-ANY annotation on that operation in addition to the NEXT annotation it already has. The resulting operation is as follows: 


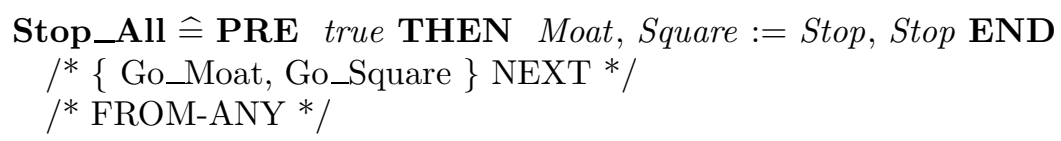

This annotation introduces an additional proof obligation. Since the precondition $P_{S t o p \_} A l l$ of Stop_All is true, it follows that $I \Rightarrow P_{S t o p \_A l l}$, and hence by Lemma 1 that the annotation is consistent with the machine.

It remains to show that the new controller Lights_CTRL2 is consistent with the annotated machine. Initial-consistency, and most of the step-consistency cases are similar to those seen in the consistency check for Lights_CTRL and we do not repeat them here. However, the new conditions are required to consider the interrupt construction $S \_I N N E R \triangle S t o p \_A l l \rightarrow S \_C T R L 2$. Step-consistency requires us to check three conditions:

1. S_INNER is step-consistent: this follows from the definition of step-consistency on process variables.

2. Stop_All $\rightarrow$ S_CTRL2 is step-consistent: this follows since S_CTRL2 is step-consistent and

$$
\begin{aligned}
\operatorname{init}\left(S \_C T R L\right) & =\{\text { Go_Moat }, \text { Go_Square, Stop_All }\} \\
& =\{\text { Go_Moat }, \text { Go_Square }\} \cup\{\text { Stop_All }\} \\
& =n \operatorname{ext}(\text { Stop_All }) \cup \text { from-any }(\text { Lights })
\end{aligned}
$$

3. $i n i t\left(S t o p \_A l l \rightarrow S \_C T R L 2\right) \subseteq$ from-any (Lights). This follows since init(Stop_All $\rightarrow$ $\left.S \_C T R L 2\right)=\left\{S t o p \_A l l\right\}$ and $S t o p \_$All has a FROM-ANY annotation.

Thus we conclude that Lights_CTRL2 is an appropriate controller for the machine Lights.

\section{Discussion}

We are currently investigating further extensions to the framework. Operations with input and output arise naturally in B machines, and can have the annotations described previously. However, the situation is more complex, since controllers can also pass information from one operation call to another. This can lead to complications in the definitions of step-consistency, and it is necessary to carry around information obtained from previous operation calls when reasoning about step-consistency. This also gives rise to parameterised recursive definitions. Query operations are of particular interest, since it appears that different considerations apply: they do not change the state of the machine, but require output which can affect control flow. 
The current approach requires separate construction of annotations and of controllers. One longer term aim of this line of research is the ability to synthesise controllers from the machine annotations. Such a controller would be the weakest controller consistent with a machine, and other consistent controllers would then be refinements. This is a topic of future research.

We now consider related work. The notion of incorporating temporal properties in B is not new. Abrial and Mussat [AM98] introduced the temporal operators of next, eventually and leadsto. In the case of leadsto (written $\rightsquigarrow$ ) they focus on identifying predicates $P$ and $Q$ such that if $P$ holds at any point then $Q$ eventually must hold, together with a list of events that make progress towards satisfying the final predicate $Q$. Their approach does not explicitly define the order in which these events must occur nor whether they occur more than once, the clause simply identifies which events can be performed in order to satisfy the $P \rightsquigarrow Q$ predicate. They use these predicates to express properties of the system which must hold when the temporal ordering of events is considered. We are using annotations to give us a handle on what operations are allowed to be performed when considering the temporal ordering of operations. We do not use them as a basis for expressing properties of a system and therefore use distinct clauses to define a possible ordering on operations in a novel way. Our approach does resonate with [AM98] in that we do not change the proof obligations that already exist but also identify additional proof obligations in order to ensure that the temporal orderings suggested by the annotations are sensible ones.

Event-B temporal operators aside, we could represent our running example in Event-B as follows:

$$
\begin{aligned}
& \text { stopall }=\text { when true then } c:=1 \text { end } \\
& \text { gosquare }=\text { when } c=1 \text { then } c:=2 \text { end } \\
& \text { stopquare }=\text { when } c=2 \text { then } c:=1 \text { end } \\
& \text { gomoat }=\text { when } c=1 \text { then } c:=3 \text { end } \\
& \text { stopmoat }=\text { when } c=3 \text { then } c:=1 \text { end }
\end{aligned}
$$

The predicates in the guards determine whether an event is enabled or not and governs when an event can be performed. In this approach the control flow is implicit and not always straightforward to understand or extract. As we saw in our example it is possible to allow either gosquare or gomoat to be performed when both variables are in the state Stop because the next annotation of the stop all operation refers to both these operations. In our approach this was translated to an external choice in CSP. In Event-B if more than one guard is true then the decision as to which event is performed is internal. Because we are only dealing temporal ordering at the level of traces this distinction is not significant. However, contrary to normal B consistency one important difference is that we do not need to examine the preconditions of all the operations to identify the next set of possible operations. By using annotations we can clearly see which operations should be available to be performed following an operation because we can look at each operation in isolation. 
The approach in [Tre00] combines CSP and B so that CSP captures, primarily, the event aspect of the design, whereas the B captures the state evolution. Each CSP controller directs a single B machine via communication channels. Controllers may also interact with other controllers. In [Tre00], consistency between the pre-conditioned B machine and the CSP controllers is established in two ways. Firstly, by showing that operations are always called within their preconditions, which establishes divergence freedom. Guarded controllers present the possibility of controller deadlock. A second, consistency condition establishes that controllers are deadlock free. Consistency is investigated using the weakest preconditions of guarded commands [Dij97], by translating the controller into AMN and demonstrating that it preserves a control loop invariant (CLI). In contrast, in this paper we establish divergence freedom by showing that the controller is consistent with the machine annotations. If every operation has a next annotation then the machine is also deadlock-free.

\section{References}

[Abr96] J-R. Abrial. The B-Book: Assigning Programs to Meaning. Cambridge University Press, 1996.

[AM98] J-R. Abrial and L. Mussat. Introducing dynamic constraints in B. In B'98, number 1393 in LNCS. Springer, 1998.

[Dij97] E. W. Dijkstra. A Discipline of Programming. Prentice Hall PTR, 1997.

[Hoa85] C.A.R. Hoare. Communicating Sequential Processes. Prentice-Hall, 1985.

[MAV05] C. Métayer, J-R. Abrial, and L. Voisin. Event-B language, 2005. RODIN deliverable 3.2, Project IST-5111599.

[MS06] A. McEwan and S. Schneider. A verified hardware development using CSP $\|$ B. In Fourth ACM-IEEE International Conference on Formal Methods and Models for Codesign, 2006.

[Sch99] S. Schneider. Concurrent and Real-time Systems: The CSP Approach. John Wiley and Sons, 1999.

[ST05] S.A. Schneider and H.E. Treharne. CSP theorems for communicating B machines. Formal Aspects of Computing, 17(4):390-422, 2005.

[Tre00] H. Treharne. Combining Control Executives and Software Specifications. PhD thesis, Royal Holloway, University of London, 2000. 